Pacific Journal of Mathematics

LOCALLY CONVEX SPACES OF NON-ARCHIMEDEAN 


\title{
LOCALLY CONVEX SPACES OF NON-ARCHIMEDEAN VALUED CONTINUOUS FUNCTIONS
}

\author{
Willy Govaerts
}

\begin{abstract}
We study the space $C(X, K, \mathcal{P})$ of all continuous functions from the ultraregular space $X$ into the non-Archimedean valued field $K$ with topology of uniform convergence on a family $\varphi$ of subsets of the Z-repletion of $X$. We characterize the bornological space associated to $C(X, K, \mathcal{P})$, semi-bornological spaces $C(X, K, \mathscr{P})$, reflexivity and semi-reflexivity both for spherically complete and non-spherically complete $K$.
\end{abstract}

1. Introduction. Throughout this paper, $K$ is a complete non-trivially non-Archimedean valued field and $X$ is an ultraregular ( $=$ zerodimensional Hausdorff) space. Then $X \subseteq v_{K} X \subseteq v_{0} X \subseteq \beta_{0} X$ where $v_{K} X$, $v_{0} X$ and $\beta_{0} X$ are the $K$-repletion, $\mathbf{Z}$-repletion and Banaschewski compactification of $X$, respectively. If $K$ has nonmeasurable cardinal, then $v_{K} X=v_{0} X[\mathbf{1}$, Theorem 15].

The set $|K|=\{|\lambda|: \lambda \in K\}$ is provided with a topology in which all points are discrete, except for 0 , whose neighborhoods are the usual ones. $|K|$ is a complete metric space under the metric

$$
d(x, y)= \begin{cases}\max (x, y) & \text { if } x \neq y, \\ 0 & \text { if } x=y .\end{cases}
$$

Hence $|K|$ is Z-replete [1, Theorem 9], so $|f|$ can be extended continuously over the whole of $v_{0} X$ whenever $f$ belongs to the vector space $C(X, K)$ of all continuous functions from $X$ into $K$.

A set $A \subseteq v_{0} X$ is called bounding if $\|f\|_{A}:=\sup _{x \in A}|f|(x)<\infty$ for all $f \in C(X, K)$. We omit the relatively easy proof of the following:

Proposition 1. The following are equivalent for $A \subseteq v_{0} X$ :

(i) $A$ is bounding.

(ii) Every $g \in C\left(v_{0} X,|K|\right)$ is bounded on $A$.

(iii) If $\left(U_{i}\right)_{l=1}^{\infty}$ is a partition of $v_{0} X$ in open-and-closed subsets, then $U_{\imath} \cap A=\varnothing$ for all but finitely many $i$.

(iv) If $g \in C\left(v_{0} X,|K|\right)$, then $g(A)$ is compact in $|K|$.

(v) If $g \in C\left(v_{0} X,|K|\right)$, then $g(A)$ is relatively compact in $|K|$.

(vi) $\bar{A}^{v_{0} X}$ is compact. 
Let $\mathscr{P}$ be an arbitrary family of subsets of $v_{0} X$ such that $Y_{\mathscr{P}}:=\cup \mathcal{P}$ is dense in $v_{0} X$. Let $C(X, K, \mathcal{P})$ be the Hausdorff locally convex space $C(X, K)$ with topology of uniform convergence on all members of $\mathcal{P}$. Without loss of generality we assume:

(i) If $A, B \in \mathscr{P}$, then $A \cup B \in \mathscr{P}$.

(ii) If $A \in \mathscr{P}, B \subset A$, then $B \in \mathscr{P}$.

(iii) If $A \in \mathscr{P}$, then $\bar{A}^{Y_{\text {i }}} \in \mathcal{P}$.

If $\mathscr{P}$ is the set $\mathscr{K}(X)$ (resp. $\mathscr{Q}(X))$ of all compact (resp. finite) subsets of $X$, then we write $C_{c}(X, K)\left(\operatorname{resp} . C_{s}(X, K)\right)$ instead of $C(X, K, \mathcal{P})$.

Definition 2. The family $\overline{\mathcal{P}}:=\left\{B \subseteq v_{0} X: \exists B^{\prime} \in \mathcal{P}\right.$ with $\left.B \subseteq \bar{B}^{\prime v_{0}} X\right\}$ is called the extended family of $\mathscr{P}$.

$\mathcal{P}$ and $\overline{\mathcal{P}}$ induce the same topology on $C(X, K) . \overline{\mathcal{P}}$ satisfies (i)-(iii) as well as

(iii)' If $A \in \overline{\mathcal{P}}$, then $\bar{A}^{v_{0} X} \in \overline{\mathcal{P}}$.

Definition 3. If $A \subseteq v_{0} X, A_{n} \subseteq v_{0} X$ for all $n=1,2, \ldots$ then $\left(A_{n}\right)_{n=1}^{\infty}$ is $A$-finite if $A_{n} \cap A=\varnothing$ for all but finitely many $n$. $\left(A_{n}\right)_{n=1}^{\infty}$ is $\mathscr{P}$-finite if it is $A$-finite for all $A \in \mathscr{P}$.

Proposition 4. Let $Y_{\circledast} \subseteq Z \subseteq v_{0} X, A \subseteq Z$. The following are equivalent:

(a) Every bounded subset of $C(X, K, \mathscr{P})$ is uniformly bounded on $A$.

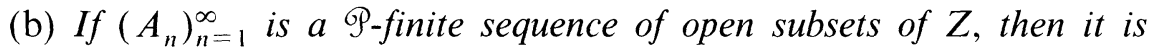
A-finite.

In (b) we can replace "open" by "clopen" and/or " $Z$ " by " $v_{0} X$ ".

Definition 5. Let $Y_{\text {oP }} \subseteq Z \subseteq v_{0} X$. The $Z$-saturated family $\tilde{\rho}^{Z}$ associated to $\mathscr{P}$ is the set of all $A \subseteq Z$ that satisfy one of the conditions mentioned in Proposition 4. $\mathscr{P}$ is $Z$-saturated iff $\tilde{\mathcal{P}} Z=\mathscr{P}$. We write $\tilde{\mathcal{P}}$ instead of $\tilde{\mathcal{\rho}} v_{0} X$.

2. Completeness and quasi-completeness. The results in this section are relatively easy and are stated here mainly for further use.

THEOREM 6. Assume $Y_{\overline{\bar{\top}}} \subseteq v_{K} X$ and let $F_{\odot \mathrm{\rho}}(X, K)$ be the set of all $f$ : $Y_{\overline{\mathrm{N}}} \rightarrow K$ that are continuous on every $A \in \overline{\mathcal{P}}$. Then:

(1) $F_{\text {vp }}(X, K)$ is a vector space over $K$ and contains $C(X, K)$ as a subspace.

(2) $F_{\text {cp }}(X, K)$ is a locally convex space under the semi-norms \|\|$_{A}$ $(A \in \overline{\mathcal{P}})$ where $\|f\|_{A}:=\sup _{x \in A}|f(x)|$. 
(3) The natural imbedding $C(X, K, \mathscr{P}) \rightarrow F_{\text {cp }}(X, K)$ is an into homeomorphism.

(4) $F_{\odot p}(X, K)$ is complete and contains $C(X, K, \mathscr{P})$ as a dense subspace; hence it is a completion of $C(X, K, \mathcal{P})$.

THEOREM 7. Assume $Y_{\overline{\text { 甲 }}} \subseteq v_{K} X$. The following are equivalent.

(1) $C(X, K, \mathscr{\rho})$ is complete.

(2) $C(X, K, \mathcal{P})$ is quasi-complete.

(3) If $f: Y_{\overline{\mathrm{\rho}}} \rightarrow K$ is continuous on every $A \in \overline{\mathcal{P}}$, then there is a $g \in C(X, K)$ such that $f(x)=g(x)$ for all $x \in Y_{\overline{\mathrm{o}}}$.

Proof. (hint for $(2) \Rightarrow(3)$ ). Let $f$ be as stated. Choose a sequence $\left(\lambda_{n}\right)_{n=1}^{\infty}$ in $K$ with $\left|\lambda_{n}\right| \underset{n \rightarrow \infty}{\rightarrow} \infty$. For all $n$ put

$$
\begin{gathered}
f_{n}(x)= \begin{cases}f(x) & \text { if }|f(x)| \leq\left|\lambda_{n}\right|, \\
\lambda_{n} & \text { if }|f(x)|>\left|\lambda_{n}\right|,\end{cases} \\
S_{n}=\left\{g \in C(X, K):|g(x)| \leq\left|\lambda_{n}\right| \text { for all } x \in X\right\} .
\end{gathered}
$$

By quasi-completeness there is a function $f_{n}^{\prime} \in C(X, K, \mathscr{P})$ with

$$
f_{n}^{\prime}(X)= \begin{cases}f(x) & \text { if }|f(x)| \leq\left|\lambda_{n}\right| \text { and } x \in Y_{\bar{p}}, \\ \lambda_{n} & \text { if }|f(x)|>\left|\lambda_{n}\right| \text { and } x \in Y_{\overline{\bar{p}}}\end{cases}
$$

Let $S=\left\{g \in C(X, K):|g(x)| \leq|f(x)|\right.$ for all $\left.x \in Y_{\overline{\text { op }}}\right\}$. By quasicompleteness, the Cauchy-net $\left(f_{n}^{\prime}\right)_{n=1}^{\infty}$ in $S$ has a limit $g$ and $g(x)=f(x)$ for all $x \in Y_{\overline{\text { p. }}}$.

REMARK 8 . For spherically complete $K$ an example of a quasi-complete, non-complete locally convex space over $K$ may be constructed as in the real case ([2, Chap. III, §2.5.]; communicated by N. De Grande-De Kimpe).

3. The bornological space associated to $C(\boldsymbol{X}, \boldsymbol{K}, \mathcal{P})$. J. Schmets [10, Théorème III.12] characterized the bornological space associated to $C(X, \mathcal{P})$, the classical (Archimedean) analogue of $C(X, K, \mathscr{P})$. We give an analogous characterization of the bornological space associated to $C(X, K, \mathscr{P})$. We assume the reader consults [10] and mainly stress the new features.

It is nice to remark that completeness of $K$ can be dispensed with in this section. 
Definition 9. If $f \in C(X, K)$, put $\Delta(f)=\{g \in C(X, K):|g| \leq|f|\}$. In particular, put $\Delta=\Delta(1)$. Let $f$ be the continuous extension of $f$ to a function $\beta_{0} X \rightarrow \beta_{0} K$.

Let $D$ be an absolutely convex absorbing subset of $C(X, K)$ such that $\Delta \subseteq D$. A compact subset $A$ of $\beta_{0} X$ is called a carrier of $D$ if $f \in D$ whenever $f$ vanishes on $A$.

The set of all carriers of $D$ will be denoted by $\mathbb{C}_{D}$.

LEMma 10. Let $A \subseteq \beta_{0} X$ be compact. If $f \in D$ whenever $f$ vanishes on a neighborhood of $A$ in $\beta_{0} X$, then $A \in \mathbb{Q}_{D}$.

Lemma 11. If $A, B \in \mathbb{Q}_{D}$, then $A \cap B \in \mathbb{Q}_{D}$.

Lemma $12 . \mathbb{Q}_{D}$ contains a smallest element $\mathscr{K}(D)$.

LEMma 13. If $\tilde{f}(x) \in \overline{\{\lambda \in K:|\lambda| \leq 1\}}^{\beta_{0} K}$ for all $x \in K(D)$, then $f \in D$.

LEMMA 14. The following are equivalent:

(a) $K(D) \subseteq v_{0} X$.

(b) $D$ is a neighborhood in $C\left(X, K, \mathcal{K}\left(v_{0} X\right)\right)$.

(c) $D$ is bornivorous in $C\left(X, K, \mathcal{K}\left(v_{0} X\right)\right)$.

The above results are in a form that make them comparable with the Archimedean ones as given in [10, Théorème III.1.2 and related results]. The Archimedean analogues go back to [9].

THEOREM 15. $C(X, K, \tilde{\mathscr{P}})$ is the bornological space associated to $C(X, K, \mathscr{P})$.

Proof. By (a) of Lemma 4 , it suffices to prove that $C(X, K, \tilde{\mathcal{P}})$ is bornological. Let $D$ be an absolutely convex bornivorous subset of $C(X, K, \tilde{\mathcal{P}})$. We may assume $\Delta \subseteq D$ (for $\Delta$ is bounded). By Lemma 14 we have $K(D) \subseteq v_{0} X$; from Lemma 13 we induce that $\{f \in C(X, K)$ : $\left.\|f\|_{K(D)} \leq 1\right\} \subseteq D$. Hence it suffices to prove that $K(D) \in \tilde{\mathcal{P}}$. Suppose not.

By Lemma 4 (b) there is a $\mathscr{P}$-finite sequence $\left(A_{n}\right)_{n=1}^{\infty}$ of open subsets of $v_{0} X$ that is not $K(D)$-finite. We may assume each $A_{n}$ to be open-andclosed and $A_{n} \cap K(D) \neq \varnothing$ for all $n$. For all $n$ there is an $f_{n} \in C(X, K)$ with $\tilde{f}_{n}=0$ on $\beta_{0} X \backslash A_{n}$ and $f_{n} \notin D$ (since $\beta_{0} X \backslash A_{n} \notin \mathbb{Q}_{D}$ ). If $\lambda_{0} \in K$, $0<\left|\lambda_{0}\right|<1$, then $\cup_{n=1}^{\infty} \Delta\left(\lambda_{0}^{-n} f_{n}\right)$ is bounded in $C(X, K, \mathscr{P})$ so there is a 
$\lambda \in K$ with $\cup_{n=1}^{\infty} \Delta\left(\lambda_{0}^{-n} f_{n}\right) \subseteq \lambda D$. Then for all $n, f_{n} \in \lambda_{0}^{n} \lambda D$, a contradiction.

Corollary 16. $([5,6])$. If $\mathscr{P}=\mathscr{K}(X)$ or $\mathscr{P}=\mathscr{Q}(X)$, then $C(X, K, \mathscr{P})$ is bornological iff $X$ is $\mathbf{Z}$-replete.

4. Semi-bornological spaces $\boldsymbol{C}(\boldsymbol{X}, \boldsymbol{K}, \mathscr{P})$ and $\boldsymbol{C}(\boldsymbol{X}, \mathscr{P})$. In this section we characterize the semi-bornological spaces $C(X, K, \mathcal{P})$ as well as their Archimedean counterparts $C(X, \mathscr{P})$. In the non-Archimedean setting semi-bornological spaces $C(X, K, \mathcal{P})$ are bornological in most practically occurring cases. In the Archimedean setting this turns out not to be true.

We use the notations of A. C. M. Van Rooij [11] on non-Archimedean measure theory. The notations on $C(X, \mathcal{P})$ are taken from J. Schmets [10]. In particular, $X$ then denotes a completely regular Hausdorff space. Our main result is the following:

THEOREM 17. If $C(X, K, \mathcal{P})$ is bornological (equivalently, $\tilde{\mathcal{P}} \subseteq \overline{\mathcal{P}}$ ), then it is semi-bornological. Conversely, assume that either $K$ is spherically complete or has non-measurable cardinality. Then, if $C(X, K, \mathscr{P})$ is semibornological, it is bornological.

Proof. We prove only the second part. Let $A \in \tilde{\mathcal{P}}$; we may assume $A$ is closed in $\bar{A}^{v_{0} X}$, i.e. $A$ is compact. From [11, Theorem 7.9] we infer that there is a non-Archimedean measure $\mu$ on $A$ such that, for every openand-closed subset $B \subseteq A$ there is an $f \in C(A, K)$ with $\|f\|_{A \backslash B}=0$ and $\int_{A} f d \mu \neq 0$. Define $L: C\left(v_{0} X, K\right) \rightarrow K$ by $L(f)=\int_{A} f d \mu$. Then $L$ is linear and $|L(f)| \leq\|f\|_{A} \cdot\|A\|_{\mu}$ for $f \in C\left(v_{0} X, K\right)$. By the assumption on $K$ in the theorem we may assume $L$ is defined on the whole of $C(X, K)$ with $|L(f)| \leq\|f\|_{A} \cdot\|A\|_{\mu}$ for all $f \in C(X, K)$.

Since $A \in \tilde{\mathcal{P}}, L$ is bounded (Lemma 4 and Definition 5) so $L$ is continuous. Let $A^{\prime} \in \mathcal{P}$ be such that $L$ is $\|\cdot\|_{A^{\prime}}$ continuous; we prove $A \subseteq{\overline{A^{\prime}}}^{v_{0} X}$.

Suppose not. Let $A^{\prime \prime}$ be an open-and-closed subset of $A \backslash A^{\prime}$. Let $f \in C(A, K)$ be zero on $A \backslash A^{\prime}$ and $\int_{A} f d \mu \neq 0$. Since $A^{\prime \prime}$ is compact, $f$ can be extended to a function in $C\left(A \cup \overline{A^{\prime} v_{0} X}, K\right)$ with $\|f\|_{A^{\prime}}=0$. By completeness of $K$ and compactness of $A \cup \overline{A^{\prime}}{ }^{{ }_{0} X} X$ it follows from [11, Theorem 5.24] that $f$ can be extended to a function in $C\left(v_{0} X, K\right)$. Hence $\|f\|_{A^{\prime}}=0$ and $L(f) \neq 0$, a contradiction.

Surprisingly, the Archimedean analogue of Theorem 17 does not hold; a more complicated theory has to be developed. We make free use of the notations in [10]. 
Definition 18. A Radon measure $\mu$ on a compact Hausdorff space $A$ is called strictly positive if $|\mu|(U) \neq 0$ for every non-empty open subset $U$ of $A$.

The strict family associated to $\mathscr{P}$ is $\mathscr{P}_{\text {str }}=\left\{A \in \mathscr{P}: \bar{A}^{v X}\right.$ carries a strictly positive measure\}.

Remark that $\mathscr{P}_{\text {str }}$ satisfies (i)-(iii) and $Y_{\mathscr{P}_{\mathrm{str}}}=Y_{\mathscr{P}}$. We have $(\alpha) \Rightarrow(\beta)$ $\Rightarrow(\gamma)$ where:

( $\alpha) A$ is separable.

( $\beta$ ) $A$ carries a strictly positive measure.

( $\gamma$ ) Every family of nonempty disjoint open subsets of $A$ is countable.

Lemma 19. Let L: $C(X, \mathcal{P}) \rightarrow \mathbf{R}$ be bounded and linear. Then it is continuous on $C\left(X,\left(\tilde{\mathscr{\rho}}^{v}\right)_{\text {str }}\right)$.

Proof. Cf. [7, Lemma 1].

Lemma 20. Assume $\mathscr{P}$ and $\mathcal{2}$ satisfy (i)-(iii) and $\mathscr{P} \subseteq \overline{\mathcal{2}}$. If $C(X, \mathcal{Q})$ is semi-bornological, then $\left(\tilde{\mathcal{P}}^{v}\right)_{\text {str }} \subseteq \overline{\mathcal{Q}}$.

Proof. Let $A \in\left(\tilde{\mathscr{\rho}}^{v}\right)_{\mathrm{str}}$. We may assume $A$ is compact. Let $\mu$ be a strictly positive measure on $A$ and put $L(f)=\int_{A} f d \mu$. Then $L$ is bounded on all $\mathscr{P}$-bounded sets.

Since $\mathscr{P} \subseteq \overline{\mathcal{Q}}$ and $C(X, \mathcal{Q})$ is semi-bornological, it follows that $L$ is continuous on $C(X, \mathcal{2})$. Let $B \in \overline{\mathcal{Q}}$ and $\varepsilon>0$ be such that $|L(f)| \leq 1$ whenever $\|f\|_{B} \leq \varepsilon$.

We may assume $B$ is compact; a standard device then shows that $A \subseteq B$. Hence $A \in \overline{\mathcal{Q}}$.

THEOREM 21. There is a smallest family 2 of relatively compact subsets of $v X$ such that (a)-(c) hold:

(a) 2 satisfies (i)-(iv).

(b) $2 \supseteq \mathscr{P}$.

(c) $C(X, 2)$ is semi-bornological.

Actually, $\mathscr{Q}=\left\{A \subseteq v X\right.$ : there exist $A_{1} \in \mathscr{P}, A_{2} \in\left(\tilde{\mathscr{\rho}}^{v}\right)_{\text {str }}$ such that $A \subset$ $\left.\overline{A_{1} \cup A_{2}}{ }^{v X}\right\}$.

Proof. From Lemmas 19 and 20.

COROLlaRY 22. $C(X, \mathcal{P})$ is semi-bornological iff $\left(\tilde{\mathscr{P}}^{v}\right)_{\mathrm{str}} \subseteq \overline{\mathcal{P}}$. 
Remarks 23. (a) Let $X$ be a compact Hausdorff space that carries no strictly positive measure and put $\mathscr{P}=(\mathscr{K}(X))_{\text {str }}$. Then $\left(\tilde{\mathscr{P}}^{v}\right)_{\text {str }} \subseteq$ $(\mathscr{K}(X))_{\text {str }}=\mathscr{P} \subseteq \overline{\mathcal{P}}$ so $C(X, \mathcal{P})$ is semi-bornological. On the other hand, $C(X, \mathscr{P})$ is not bornological; in fact $C_{c}(X)$ is the bornological space associated to $C(X, \mathcal{P})$.

(b) Clearly $\mathscr{P} \subseteq \overline{\mathcal{P}} \subseteq \tilde{\mathcal{P}}^{v}$ and $\mathscr{P}_{\text {str }} \subseteq \mathscr{P}$. Less trivially we have $\left(\mathscr{P}_{\text {str }}\right)^{v} \supseteq$ $\tilde{\mathscr{P}}^{v}$; this is proved by an argument involving the fact that separable members of $\mathscr{P}$ belong to $\mathscr{P}_{\text {str }}$.

THEOREM 24. Let $\mathscr{P}=\mathscr{K}(X)$ or $\mathscr{P}=\mathbb{Q}(X)$. The following are equivalent:

(1) $C(X, \mathscr{P})$ is bornological.

(2) $C(X, \mathcal{P})$ is semi-bornological.

(3) $X=v X$.

Proof. The equivalence of (1) and (3) is known ([10]), while (1) $\Rightarrow(2)$ is trivial.

To prove $(2) \Rightarrow(3)$ remark that $\mathscr{Q}(X)^{v}=\mathscr{Q}(v X)$ ([10, III.4.3]) so $\left(\mathscr{Q}(X)^{v}\right)_{\mathrm{str}}=\mathscr{Q}(v X)$. If $\mathscr{P}=\mathscr{K}(X)$, see [10, III.2.4].

5. Reflexivity and semi-reflexivity for non spherically complete $K$. In this section we assume $Y_{\bar{\Phi}} \subseteq v_{K} X$. Since $Y_{\bar{\Phi}}$ is dense in $v_{0} X$ the dual $C(X, K, \mathcal{P})^{\prime}$ of $C(X, K, \mathscr{P})$ separates the points of $C(X, K, \mathscr{P})$. Let $b$ be the strong topology on $C(X, K, \mathcal{P})^{\prime}$. There is a natural injection from $C(X, K, \mathcal{P})$ into $\left(C(X, K, \mathcal{P})_{b}^{\prime}\right)^{\prime} . C(X, K, \mathcal{P})$ is called semi-reflexive if this injection is onto, and reflexive if it is a homeomorphism onto $\left(C(X, K, \mathscr{P})_{b}^{\prime}\right)_{b}^{\prime}$.

Lemma 25. If $L \in C(X, K, \mathcal{P})^{\prime}$, then there is a compact subset $A_{L} \subset v_{0} X$ such that:

(1) For all $\varepsilon>0$ there is a $\delta>0$ such that $|L(f)| \leq \varepsilon$ whenever $f \in C(X, K)$ and $\|f\|_{A_{L}} \leq \delta$.

(2) If $A$ is a compact subset of $v_{0} X$ and $L$ is bounded on $\|\cdot\|_{A}$-bounded subsets of $C(X, K)$, then $A_{L} \subset A$.

(3) If $A$ is a compact subset of $v_{0} X$ and $L(f)=0$ for all $f \in C(X, K)$ for which $\|f\|_{A}=0$, then $A_{L} \subset A$.

(4) If $f \in C(X, K)$ and $\|f\|_{A_{L}}=0$, then $L(f)=0$.

(5) $A_{L} \in \overline{\mathcal{P}}$.

The set $A_{L}$ is called the carrier of $L$. 
REMARK 26. By [11, Theorem 7.18] there is a non-Archimedean measure $\mu$ on $A_{L}$ such that $L(f)=\int_{A_{L}} f d \mu$ for all $f \in C(X, K)$.

COROllaRy 27. Let $\mathscr{P}$ be directed by $\leq$ where $A \leq B$ iff $\bar{A}^{v_{0} X} \subseteq{\overline{B^{v}}}^{v_{0}}$. If $A \leq B$ put

$$
h_{B, A}: C_{c}\left(\bar{A}^{v_{0} X}, K\right)^{\prime} \rightarrow C_{c}\left(\bar{B}^{v_{0} X}, K\right)^{\prime},
$$

where $\left(h_{B, A}(L)\right)(f)=L\left(\left.f\right|_{A^{v_{0}} X}\right)$ whenever $L \in C_{c}\left(\bar{A}^{v_{0} X}, K\right)^{\prime}$ and $f \in$ $C_{c}\left(\bar{B}^{v_{0} X}, K\right)$.

For $A \in \mathcal{P}$ put

$$
h_{A}: C_{c}\left({\overline{A^{v}}}^{v_{0}}, K\right)^{\prime} \rightarrow C(X, K, \mathscr{P})^{\prime}
$$

where $\left(h_{A}(L)\right)(f)=L\left(f \mid \bar{A}_{0} x\right)$ whenever $L \in C\left(\bar{A}^{v_{0} X}, K\right)^{\prime}$ and $f \in$ $C(X, K)$. Then $C(X, K, \mathscr{P})^{\prime}$ is the algebraic inductive limit of $\left(C_{c}\left(\bar{A}^{v_{0} X}, K\right)^{\prime}\right)_{A \in \mathcal{Q P}}$ with respect to the above $h_{B, A}$ and $h_{A}$.

Let $B$ be bounded in $C(X, K, \mathcal{P}), B_{A}(A \in \mathcal{P})$ the set of all restrictions to $\bar{A}^{v_{0} X}$ of functions from $B$. Every $B_{A}(A \in \mathcal{P})$ is bounded in $C_{c}\left(\bar{A}^{v_{0}} X, K\right)$ and

$$
B^{0}=\bigcup_{A \in \mathscr{P}} h_{A}\left(\left(B_{A}\right)^{0}\right)
$$

where $B^{0}$ (resp. $\left.B_{A}^{0}\right)$ is the polar of $B$ (resp. $\left.B_{A}\right)$ in $C(X, K, \mathscr{P})^{\prime}$ (resp. $\left.C_{c}\left(\bar{A}^{v_{0}} X, K\right)^{\prime}\right)$.

Lemma 28. Let $\varphi \in\left(C(X, K, \mathcal{P})_{b}^{\prime}\right)^{\prime}$. For every $A \in \mathcal{P}$ there is a $\varphi_{A} \in C_{c}\left(\bar{A}^{v_{0} X}, K\right)^{\prime \prime}$ such that

$$
\varphi\left(h_{A}(L)\right)=\varphi_{A}(L) \quad \text { for all } L \in C_{c}\left({\overline{A^{v}}}^{v_{0}}, K\right)^{\prime} .
$$

Proof. By assumption there is a bounded set $B$ in $C(X, K, \mathcal{P})$ such that $\left|\varphi\left(L_{0}\right)\right| \leq 1$ whenever $L_{0} \in B^{0}$. If $A \in \mathcal{P}$, then $\left|\varphi\left(h_{A}(L)\right)\right| \leq 1$ whenever $L \in B_{A}^{0}$. Since $\varphi \circ h_{A}$ is linear, we infer $\varphi \circ h_{A} \in C_{c}\left(\bar{A}^{v_{0} X}, K\right)^{\prime \prime}$.

Proposition 29. Assume $K$ non-spherically complete, $K$ and $Y$ nonmeasurable, $\varphi \in\left(C(X, K, \mathcal{P})_{b}^{\prime}\right)^{\prime}$. Then there is a function $f: Y_{\bar{\top}} \rightarrow K$ such that:

(1) $\left.f\right|_{A}$ is continuous on every $A \in \overline{\mathcal{P}}$.

(2) $\varphi\left(h_{A}(L)\right)=L\left(\left.f\right|_{A}\right)$ for all $A \in \overline{\mathcal{P}}$ and $L \in C_{c}\left(\bar{A}^{v_{0} X}, K\right)^{\prime}$.

Proof. For every $A \in \mathscr{P}$ the space $C_{c}\left(\bar{A}^{v_{0} X}, K\right)$ is isomorphic to a space of type $c_{0}(I)[11,5.23]$. Since $K$ and $X$ are nonmeasurable, $I$ is 
nonmeasurable. By [11, Theorem 4.21] $c_{0}(I)$ is reflexive. Hence there is an $f_{A} \in C_{c}\left(\bar{A}^{v_{0} X}, K\right)$ such that $\varphi\left(h_{A}(L)\right)=L\left(f_{A}\right)$ for all $L \in C_{c}\left(\bar{A}^{v_{0} X}, K\right)^{\prime}$.

Let $A, B \in \mathcal{P}, A \leq B$. For $L \in C_{c}\left(\bar{A}^{v_{0} X}, K\right)^{\prime}$ we have

$$
L\left(f_{A}\right)=\varphi\left(h_{A}(L)\right)=\varphi\left(h_{B}\left(h_{B, A}(L)\right)\right)=\left(h_{B, A}(L)\right)\left(f_{B}\right) .
$$

In particular, if $L=\delta_{a}\left(a \in \vec{A}^{\nu_{0} x}, \delta_{a}\right.$ the evaluation in $\left.a\right)$, then

$$
f_{A}(a)=\delta_{a}\left(f_{A}\right)=\left(f_{B, A}\left(\delta_{a}\right)\right)\left(f_{B}\right)=\delta_{a}\left(f_{B}\right)=f_{B}(a) .
$$

Hence there is an $f: Y \rightarrow K$ such that $\left.f\right|_{A^{v_{0}} x}=f_{A}$ for all $A \in \mathcal{P}$; clearly (1) and (2) hold.

TheORem 30. Assume $K$ non-spherically complete, $K$ and $X$ nonmeasurable. The following are equivalent:

(1) $C(X, K, \mathcal{P})$ is complete.

(2) $C(X, K, \rho)$ is quasi-complete.

(3) $C(X, K, \mathcal{P})$ is semi-reflexive.

(4) If $f: Y_{\overline{\mathcal{P}}} \rightarrow K$ is continuous on every $A \in \overline{\mathcal{P}}$, then there is a $g \in C(X, K)$ such that $g=f$ on $Y_{\bar{\oplus}}$.

Proof. By the assumptions $v_{K} X=v_{0} X$. The equivalence of (1), (2) and (4) is Theorem 7. Furthermore (3) $\Rightarrow(2)$ by a standard argument (remark that $C(X, K, \mathcal{P})^{\prime}$ separates the points of the completion of $C(X, K, \mathcal{P})$ by virtue of Theorem 6$)$.

To prove that (4) $\Rightarrow(3)$ assume $\varphi \in\left(C(X, K, \mathcal{P})_{b}^{\prime}\right)^{\prime}$. Let $f$ be as in Proposition 29. By (4) there is a $g \in C(X, K)$ such that $g(x)=f(x)$ for all $x \in Y_{\overline{\mathcal{S}}}$. Let $\varphi_{g}: C(X, K, \mathscr{P})^{\prime} \rightarrow K$ be defined by $\varphi_{g}\left(h_{A}(L)\right)=L\left(\left.g\right|_{A}\right)$ for $A \in \overline{\mathscr{P}}$ and $L \in C\left(\bar{A}^{v_{0}} X, K\right)^{\prime}$. Then $\varphi=\varphi_{g}$ so $C(X, K, \mathscr{P})$ is semi-reflexive.

Theorem 31. Assume $K$ non-spherically complete, $K$ and $X$ nonmeasurable. Then $C(X, K, \mathcal{P})$ is reflexive iff both (a) and (b) hold:

(a) $C(X, K, \mathscr{P})$ is semi-reflexive.

(b) $\tilde{\mathcal{P}}{ }^{Y \bar{P}} \subset \overline{\mathcal{P}}$.

Proof. If $C(X, K, \mathcal{P})$ is reflexive, then we prove (b). Suppose $A \in \tilde{\mathscr{P}}^{Y^{\bar{\oplus}}}$ and put $B_{A}=\left\{f \in C(X, K):\|f\|_{A} \leq 1\right\}$. Let $B_{A}^{0}$ be the polar of $B_{A}$ in $C(X, K, \mathscr{P})^{\prime}$ and $B_{A}^{00}$ the bipolar in $C(X, K, \mathcal{P})$. A routine argument shows that $B_{A}^{00}=B_{A}$. If $C$ is any bounded set in $C(X, K, \mathscr{P})$, then, by Proposition 4 , there is a $\lambda \in K \backslash\{0\}$ such that $C \subseteq \lambda B_{A}$, so $B_{A}^{0} \subseteq \lambda C^{0}$; this proves $B_{A}^{0}$ is bounded in $C(X, K, \mathscr{P})_{b}^{\prime}$. By reflexivity, $B_{A}=B_{A}^{00}$ is a neighbourhood in $C(X, K, \mathscr{P})$ which implies $A \in \overline{\mathcal{P}}$. 
Now assume (a) and (b) and let $B$ be bounded in $C(X, K, \mathscr{P})_{b}^{\prime}$. Then $B^{0}$ is absolutely convex and absorbs all bounded sets, hence is a neighbourhood in the bornological space associated to $C(X, K, \mathcal{P})$, i.e. in $C(X, K, \tilde{\mathscr{P}})$ (Theorem 15). Let $A \in \tilde{\mathscr{P}}$ and $\lambda \in K \backslash\{0\}$ be such that $\left\{f \in C(X, K):\|f\|_{A} \leq|\lambda|\right\} \subseteq B^{0}$. Let $A^{\prime}=A \cap Y_{\overline{\mathcal{P}}}$. If $L \in B$ and $f \in$ $C(X, K),\|f\|_{A}=0$, then $L(f)=0$; hence $A_{L} \subseteq A$ by Lemma 25 . On the other hand $A_{L} \subseteq Y_{\overline{\mathcal{P}}}$, so $A_{L} \subseteq A^{\prime}$.

Let $f \in C(X, K)$ be arbitrary with $\|f\|_{A^{\prime}} \leq|\lambda|$. Let $g \in C(X, K)$ be such that $\|g\|_{A} \leq|\lambda|$ and $f=g$ on $A^{\prime}$. Then for $L \in B$ we have

$$
|L(f)| \leq \max (|L(g)|,|L(f-g)|) \text {. }
$$

Since $\left.f\right|_{A_{I}}=\left.g\right|_{A_{L}}, L(f-g)=0$, so $|L(f)| \leq|L(g)| \leq 1$. Now $\{f \in$ $\left.C(X, K):\|f\|_{A^{\prime}} \leq|\lambda|\right\} \subseteq B^{0}$ and $A^{\prime} \in \tilde{\mathscr{P}}^{\gamma^{\overline{\widehat{T}}}} \subseteq \overline{\mathcal{P}}$, so $B^{0}$ is a neighbourhood in $C(X, K, \mathscr{P})$.

REMARK 32. Two counterexamples prove that (a) and (b) of Theorem 31 are independent.

(1) Let $X=[0, \Omega[$, the first uncountable ordinal, $\mathscr{P}=\mathscr{K}(X)$. By Theorem 7 and local compactness of $X, C_{c}(X, K)$ is complete. However, $\tilde{\mathscr{P}}=\mathscr{K}([0, \Omega])$ and so $\left[0, \Omega\left[\in \tilde{\mathscr{P}}^{Y^{\bar{\Phi}}} \backslash \overline{\mathcal{P}}\right.\right.$.

(2) Let $X=[0, \Omega], \mathscr{P}=\mathscr{Q}(X)$. Then $\tilde{\mathscr{P}}^{Y \overline{\mathcal{P}}}=\tilde{\mathscr{P}}=\mathscr{P}=\overline{\mathscr{P}}$ (Corollary 16). However, $X$ is not discrete and so condition (3) of Theorem 7 is not fulfilled.

6. Reflexivity and semi-reflexivity for spherically complete $K$. In this section we assume $Y_{\bar{\top}} \subseteq v_{K} X$. A locally convex space over the spherically complete field $K$ is $c$-Montel if it is a barrelled space in which all absolutely convex, closed, bounded sets are $c$-compact [12, Definition 3.8.b]. From [3, Proposition 2] it follows that " $c$-Montel" is equivalent with "Montel" if $K$ is a local field.

Our main result is the following substantial generalization of [4, Theorem III.45] (See also [13]).

THEOREM 33. Let $K$ be spherically complete. The following are equivalent:

(1) $C(X, K, \mathscr{P})$ is a $c$-Montel space.

(2) $C(X, K, \mathscr{P})$ is reflexive.

(3) $C(X, K, \mathcal{P})$ is semi-reflexive.

(4) For every $f: Y_{\bar{\Phi}} \rightarrow K$ there is a $g \in C(X, K)$ such that $f=g$ on $Y_{\bar{\Phi} \text {. }}$

Proof. (1) $\Rightarrow$ (2). See [12, Corollaire 1 of Théorème 4.28].

(2) $\Rightarrow(3)$. Trivial. 
(3) $\Rightarrow(4)$. First we show that every $A \in \mathcal{P}$ is finite. If not, then there is a sequence $\left(x_{n}\right)_{n=1}^{\infty}$ in $v_{K} X$ and $x \in v_{K} X$ such that $x_{n} \neq x$ for all $n$ and $x \in\left\{x_{1}, x_{2}, \ldots\right\}^{v_{0} X}$. For all $n$ choose $f_{n} \in C(X, K)$ so that $f_{n}\left(x_{i}\right)=1$ if $i \leq n, f_{n}\left(x_{i}\right)=0$ if $i>n$, and $\left|f_{n}(y)\right| \leq 1$ for all $y \in X$.

Let $B_{n}$ be the convex hull of $\left\{f_{n}, f_{n+1}, \ldots\right\}$. The set $\{f \in C(X, K)$ : $|f(y)| \leq 1$ for all $y \in X\}$ is absolutely convex, weakly bounded and weakly closed in a semi-reflexive space, hence weakly $c$-compact [12, Théorème 4.25(2)].

Let $f$ be a weak adherence point of the convex filter $\left(B_{n}\right)_{n=1}^{\infty}$. Let $n$ be arbitrary. If $i \leq n$ and $g \in B_{n}$, then $g\left(x_{i}\right)=1$; hence $f\left(x_{i}\right)=1$. Since $f\left(x_{i}\right)=1$ for all $i$, we have $f(x)=1$. On the other hand, $g(x)=0$ for all $n \in N$ and $g \in B_{n}$; hence $f(x)=0$, a contradiction.

Since every $A \in \mathscr{P}$ is finite, $\mathscr{P}$ is the family of finite subsets of $Y_{\mathscr{P}}$. Let $T$ be an arbitrary bounded closed subset of $C(X, K, \mathcal{P})$ and $\operatorname{Co}(T)$ its absolutely convex closed hull. By [12, Théorème $\left.4.25,2^{\circ}\right] \mathrm{Co}(T)$ is weakly c-compact. From [3, Proposition 3(a)] and [8, §5, Proposition 4] we infer that $\operatorname{Co}(T)$ is $c$-compact; by [3, Proposition 1] $\operatorname{Co}(T)$ is complete. As a closed subset of a complete set, $T$ is complete.

We conclude that $C(X, K, \mathscr{P})$ is quasi-complete, hence complete. Since every $f: Y_{\overline{\mathcal{P}}} \rightarrow K$ may be pointwisely approximated by functions from $C\left(v_{k} X, K\right)$, (4) follows.

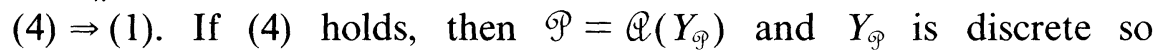
$C(X, K, \mathscr{P})$ can be identified with $K^{Y_{\text {* }}}$. Since $K$ is a $c$-Montel space, the result follows as in [4, Theorem III.45].

COROLlaRY 34. If $K$ is spherically complete and $Y_{\bar{\Phi}}=X$, then the following are equivalent:

(1) $C(X, K, \mathcal{P})$ is a c-Montel space (reflexive, semi-reflexive).

(2) $C_{c}(X, K)$ is a c-Montel space (reflexive, semi-reflexive).

(3) $C_{s}(X, K)$ is a $c$-Montel space (reflexive, semi-reflexive).

(4) $X$ is discrete.

ReMARK 35. If $K$ is spherically complete, $X=[0, \Omega]$, then $C_{c}(X, K)$ is complete (Theorem 7) but not semi-reflexive (Theorem 33).

\section{REFERENCES}

[1] G. Bachman, E. Beckenstein, L. Narici and S. Warner, Rings of continuous functions with values in a topological field, Trans. Amer. Math. Soc., 204 (1975), 91-112.

[2] N. Bourbaki, Eléments de mathématique, Livre V, Espaces vectoriels topologiques.

[3] N. De Grande-De Kimpe, c-Compactness in locally K-convex spaces, Proc. Kon. Ned. Akad. Wet., A 74 (1971), 176-180. 
[4] R. L. Ellis, Topological vector spaces over non-Archimedean fields, $\mathrm{Ph}$.D. Dissertation, Duke University, N.C. 1966.

[5] W. Govaerts, Bornological spaces of non-Archimedean valued functions with the point-open-topology, Proc. Amer. Math. Soc., 72 (1978), 571-575.

[6] B_ Bornological spaces of non-Archimedean valued functions with the compact-open topology, Proc. Amer. Math. Soc., 78 (1980), 132-134.

[7] Sequentially continuous linear functionals on spaces of continuous functions, (to appear).

[8] L. Gruson, Théorie de Fredholm p-adique, Bull. Soc. Math. de France, 94 (1966), 67-95.

[9] L. Nachbin, Topological vector spaces of continuous functions, Proc. Nat. Acad. U.S.A., 40 (1954), $471-474$.

[10] J. Schmets, Espaces de Fonctions Continues, Lecture Notes in Mathematics 519, Springer-Verlag, Berlin, 1976.

[11] A. C. M. Von Rooij, Non-Archimedean Functional Analysis, Marcel Dekker Inc., New York, 1978.

[12] J. Van Tiel, Espaces localement K-convexes I-III, Proc. Kon. Ned. Akad. Wet., 68 (1965), 249-258; 259-272; 273-289.

[13] S. Warner, The topology of compact convergence on continuous function spaces, Duke Math. J., 25 (1958), 265-282.

Received October 8, 1981 and in revised form October 15, 1982. The contents of this paper are part of the thesis "Locally convex spaces of continuous functions" that we presented to the University of Ghent in September 1980.

Seminarie Voor Hogere ANAlyse

GALGLAAN 2

B-9000 GENT

BELGIUM 


\title{
PACIFIC JOURNAL OF MATHEMATICS EDITORS
}

\author{
Donald BabBitT (Managing Editor) \\ University of California \\ Los Angeles, CA 90024 \\ Hugo RossI \\ University of Utah \\ Salt Lake City, UT 84112 \\ C. C. Moore and Arthur Ogus \\ University of California \\ Berkeley, CA 94720
}

J. DugundiI

Department of Mathematics

University of Southern California

Los Angeles, CA 90089-1113

R. FinN and H. SAMELSON

Stanford University

Stanford, CA 94305

\section{ASSOCIATE EDITORS}
R. ARENS
E. F. BECKENBACH
B. H. NeUmanN
F. WOLF
K. YosHida
(1906-1982)

\section{SUPPORTING INSTITUTIONS}

\author{
UNIVERSITY OF ARIZONA \\ UNIVERSITY OF BRITISH COLUMBIA \\ CALIFORNIA INSTITUTE OF TECHNOLOGY \\ UNIVERSITY OF CALIFORNIA \\ MONTANA STATE UNIVERSITY \\ UNIVERSITY OF NEVADA, RENO \\ NEW MEXICO STATE UNIVERSITY \\ OREGON STATE UNIVERSITY
}

\author{
UNIVERSITY OF OREGON \\ UNIVERSITY OF SOUTHERN CALIFORNIA \\ STANFORD UNIVERSITY \\ UNIVERSITY OF HAWAII \\ UNIVERSITY OF TOKYO \\ UNIVERSITY OF UTAH \\ WASHINGTON STATE UNIVERSITY \\ UNIVERSITY OF WASHINGTON
}

The Supporting Institutions listed above contribute to the cost of publication of this Journal, but they are not owners or publishers and have no responsibility for its content or policies.

Mathematical papers intended for publication in the Pacific Journal of Mathematics should be in typed form or offset-reproduced (not dittoed), double spaced with large margins. Please do not use built up fractions in the text of the manuscript. However, you may use them in the displayed equations. Underline Greek letters in red, German in green, and script in blue. The first paragraph must be capable of being used separately as a synopsis of the entire paper. In particular it should contain no bibliographic references. Please propose a heading for the odd numbered pages of less than 35 characters. Manuscripts, in triplicate, may be sent to any one of the editors. Please classify according to the scheme of Math. Reviews, Index to Vol. 39. Supply name and address of author to whom proofs should be sent. All other communications should be addressed to the managing editor, or Elaine Barth, University of California, Los Angeles, California 90024.

There are page-charges associated with articles appearing in the Pacific Journal of Mathematics. These charges are expected to be paid by the author's University, Government Agency or Company. If the author or authors do not have access to such Institutional support these charges are waived. Single authors will receive 50 free reprints; joint authors will receive a total of 100 free reprints. Additional copies may be obtained at cost in multiples of 50 .

The Pacific Journal of Mathematics is issued monthly as of January 1966. Regular subscription rate: $\$ 132.00$ a year (6 Vol., 12 issues). Special rate: $\$ 66.00$ a year to individual members of supporting institutions.

Subscriptions, orders for numbers issued in the last three calendar years, and changes of address should be sent to Pacific Journal of Mathematics, P.O. Box 969, Carmel Valley, CA 93924, U.S.A. Old back numbers obtainable from Kraus Periodicals Co., Route 100, Millwood, NY 10546.

The Pacific Journal of Mathematics ISSN $0030-8730$ is published monthly by the Pacific Journal of Mathematics at P.O. Box 969, Carmel Valley, CA 93924. Application to mail at Second-class postage rates is pending at Carmel Valley, California, and additional mailing offices. Postmaster: Send address changes to Pacific Journal of Mathematics, P. O. Box 969, Carmel Valley, CA 93924.

PUBLISHED BY PACIFIC JOURNAL OF MATHEMATICS, A NON-PROFIT CORPORATION

Copyright $\odot 1983$ by Pacific Journal of Mathematics 


\section{Pacific Journal of Mathematics}

Vol. 109, No. $2 \quad$ June, 1983

Tibor Bisztriczky, On the singularities of almost-simple plane curves . . . . 257

Peter B. Borwein, On Sylvester's problem and Haar spaces .............. 275

Emilio Bujalance, Cyclic groups of automorphisms of compact nonorientable Klein surfaces without boundary ............... 279

Robert Jay Daverman and John J. Walsh, Acyclic decompositions of

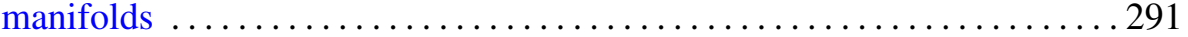

Lester Eli Dubins, Bernstein-like polynomial approximation in higher

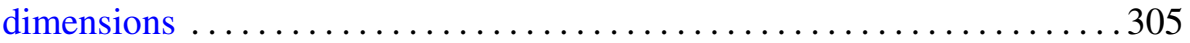

Allan L. Edelson and Jerry Dee Schuur, Nonoscillatory solutions of

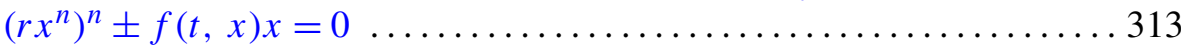

Akira Endô, On units of pure quartic number fields ................. 327

Hector O. Fattorini, A note on fractional derivatives of semigroups and

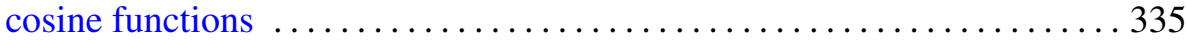

Ronald Fintushel and Peter Sie Pao, Circle actions on homotopy spheres with codimension 4 fixed point set ........................ 349

Stephen Michael Gagola, Jr., Characters vanishing on all but two

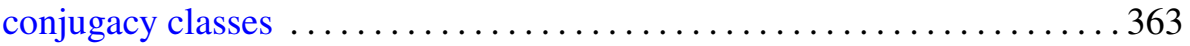

Saverio Giulini, Singular characters and their $L^{p}$ norms on classical Lie

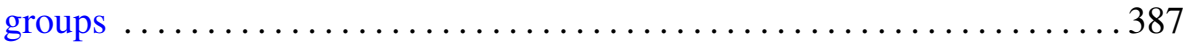

Willy Govaerts, Locally convex spaces of non-Archimedean valued continuous functions

Wu-Chung Hsiang and Bjørn Jahren, A remark on the isotopy classes of diffeomorphisms of lens spaces $\ldots . \ldots \ldots \ldots \ldots \ldots$

Hae Soo Oh, Compact connected Lie groups acting on simply connected 4-manifolds

Frank Okoh and Frank A. Zorzitto, Subsystems of the polynomial system

Knut Øyma, An interpolation theorem for $H_{E}^{\infty}$

Nikolaos S. Papageorgiou, Nonsmooth analysis on partially ordered vector spaces. II. Nonconvex case, Clarke's theory ........ 